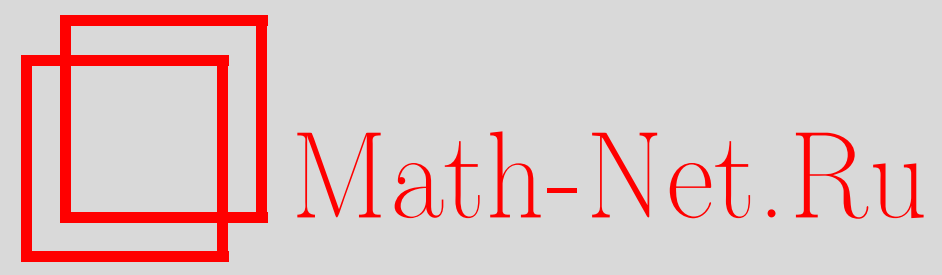

Y. Ouknine, M. Erraoui, Transformations of Two Independent Brownian Motions and Orthogonal Decompositions of Brownian Filtrations, Теория вероятн. и ее примен., 2008, том 53, выпуск 4, 769-786

DOI: https://doi.org/10.4213/tvp2464

Использование Общероссийского математического портала Math-Net.Ru подразумевает, что вы прочитали и согласны с пользовательским соглашением

http://www . mathnet.ru/rus/agreement

Параметры загрузки:

IP : 54.166 .219 .16

26 апреля 2023 г., 08:50:13

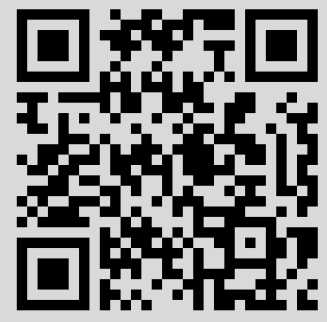




\title{
TRANSFORMATIONS OF TWO INDEPENDENT BROWNIAN MOTIONS AND ORTHOGONAL DECOMPOSITIONS OF BROWNIAN FILTRATIONS
}

\begin{abstract}
Рассматриваются некоторые классы преобразований двух независимых броуновских движений. Приводится новое ортогональное разложение некоторых броуновских фильтраций.
\end{abstract}

Ключевые слова и фразы: броуновское движение, броуновские фильтрации, каноническое разложение, операторы Харди.

1. Introduction. Motivated by the Kyle-Back model of «insider trading», Föllmer, Wu, and Yor [8], [19] investigate some mathematical problems which appear in this context. We will give a brief outline of [19]: Wu and Yor consider certain classes of linear transformations of two independent Brownian motions and study their canonical decompositions. More precisely, let $\left(B_{t}\right)_{t \geqslant 0}$ and $\left(\widetilde{B}_{t}\right)_{t \geqslant 0}$ be two independent Brownian motions defined on a complete probability space $(\Omega, \mathscr{F}, \mathbf{P})$. Consider semimartingales of the form

$$
d X_{t}=d B_{t}+Y_{t} d t
$$

where $Y$ is linear in $B$ and $\widetilde{B}$, and investigate when $X$ is again a Brownian motion relative to its own filtration. Firstly, they consider the special case

$$
Y_{t}=f(t) \widetilde{B}_{t}+g(t) B_{t},
$$

where $f$ and $g$ are two continuous functions satisfying some integral conditions, and obtain the following characterization: $X_{t}$ is a Brownian motion if and only if $f(t)= \pm \sqrt{\nu-\nu^{2}} / t$ and $g(t)=-\nu / t$ for some $\nu \in[0,1]$. This study is an extension, to the case of two independent Brownian motions, of some results about noncanonical representations of Brownian motion of [6] (for $f(t) \equiv 0$ and $g(t)=-1 / t$ ) and [11] (for $f(t) \equiv 0$ and $g(t)=-\nu / t$ ). Secondly, the authors consider the case, where $Y$ depends linearly on $X$ and $\widetilde{B}$, and characterize Brownian motions which are solutions of stochastic differential equation of the form

$$
X_{t}=B_{t}+\int_{0}^{t}\left(f(u) \widetilde{B}_{u}+g(u) X_{u}\right) d u
$$

* Faculté des Sciences Semlalia, Département de Mathématiques, Université Cadi Ayyad, BP 2390, Marrakech, Maroc; e-mail: ouknine@ucam.uc.ma, erraoui@ucam.uc.ma 
with $X_{0}=0$. In addition, they construct another Brownian motion in the natural filtration of $B$ and $\widetilde{B}$, which is independent of $X$. Using the transformation $T(B)_{t}:=B_{t}-\int_{0}^{t} u^{-1} B_{u} d u$, which leaves invariant the Wiener measure, they get two sequences of Brownian motions which are independent of each other. This enables them to deduce a new orthogonal decomposition of Brownian filtrations. Now we give two general observations. First in order to characterize Brownian motions of the form (1) the approach used is based on the resolution of linear stochastic differential equation. This kind of equations comes from the theory of enlargement of filtrations (see [10], [11], [20], and [21]). Next, the orthogonal decomposition of the Brownian filtration is in fact related to the problem of constructions of noncanonical representations of Brownian motions which was first introduced by Lévy [17] and later developed by Hida [16] and Cramér [5]. This problem, which was studied extensively within the framework of stochastic filtering, belongs to the theory of impoverishment of filtrations. Recently, Hibino, Hitsuda, and Muraoka [14] obtained a unified expression of the Lévy examples by the use of an integral operator $K_{\mathbf{g}}$ associated with a singular Volterra kernel and gave a general method to obtain a noncanonical representation of Brownian motion. This approach has its own interest in connection with a generalized Hardy inequality. We notice also that the transformation $T$ is closely related to the Hardy transform (see [20]). It is therefore interesting to explore the same questions as that in [19] from this point of view.

The paper is organized as follows.

In Section 2, in order to prepare our analysis, we introduce the definition of the Volterra operator $K_{\mathbf{g}}$ and its adjoint $K_{\mathbf{g}}^{*}$ and provide some additional properties which will be useful in the rest of the paper. In Section 3, as in [19] we construct, with the help of the operator $\left(I-\lambda K_{\mathrm{g}}^{*}\right)$, two independent Brownian motions $X$ and $Y$ in the natural filtration of $B$ and $\widetilde{B}$ having a noncanonical Volterra representation (i.e., $\mathscr{F}_{t}^{(X, Y)} \subsetneq \mathscr{F}_{t}^{(B, \widetilde{B})}$ ). In Section 4, by using the method of iteration we get two new sequences of Brownian motions which are independent of each other that enables us to construct a new orthogonal decomposition of the filtration generated by $X$ and $Y$.

2. Generalized Hardy operators. At the beginning, let us recall the Hardy transform given by

$$
H: L^{2}[0,1] \rightarrow L^{2}[0,1], \quad f \mapsto H f: t \rightarrow \frac{1}{t} \int_{0}^{t} f(s) d s,
$$

The adjoint of $H$, which we denote by $\tilde{H}$, is given by

$$
\widetilde{H}(f)(t)=\int_{t}^{1} \frac{f(s)}{s} d s, \quad f \in L^{2}([0,1]) .
$$


It is well known that the operator $K=H$ or $\widetilde{H}$ satisfies Hardy's $L^{2}$ inequality:

$$
\left(\int_{0}^{1}(K f)^{2}(s) d s\right)^{1 / 2} \leqslant 2\left(\int_{0}^{1} f^{2}(s) d s\right)^{1 / 2} .
$$

Let $\mathbf{g}=\left(g_{1}, \ldots, g_{N}\right), g_{i} \in L^{2}[0,1], i=1, \ldots, N$, be a linearly independent system. We define the operator $K_{\mathbf{g}}$ on $L^{2}([0,1])$ by

$$
\left(K_{\mathbf{g}} \alpha\right)(t)=\mathbf{g}^{\tau}(t) G^{-1}(t) \int_{0}^{t} \mathbf{g}(s) \alpha(s) d s,
$$

where

$$
\begin{aligned}
\mathbf{g}^{\tau}(t) & =\left(g_{1}(t), \ldots, g_{N}(t)\right)^{\tau} \\
G(t) & =\int_{0}^{t} \mathbf{g}^{\tau}(s) \mathbf{g}(s) d s=\left(\int_{0}^{t} g_{i}(s) g_{j}(s) d s\right)_{i, j=1, \ldots, N}, \\
\int_{0}^{t} \mathbf{g}(s) \alpha(s) d s & =\left(\int_{0}^{t} g_{1}(s) \alpha(s) d s, \ldots, \int_{0}^{t} g_{N}(s) \alpha(s) d s\right)^{\tau} .
\end{aligned}
$$

Here $\mathbf{g}^{\tau}$ stands for the transpose of $\mathbf{g}$ and $G(t)$ is the Grammian matrix of $\mathrm{g}$ and $G^{-1}(t)$ its inverse matrix. Here we note that $G^{-1}(t)$ exists, since $g_{i}$ 's are linearly independent.

It is well known (see [14]) that $K_{\mathbf{g}}$ is a bounded operator and satisfies

$$
\left\|K_{\mathbf{g}} \alpha\right\| \leqslant 2\|\alpha\| \quad \forall \alpha \in L^{2}([0,1]),
$$

and

$$
\operatorname{Ker}\left(I-K_{\mathbf{g}}\right)=\operatorname{LS}\left\{g_{1}, \ldots, g_{N}\right\},
$$

where $\operatorname{LS}\left\{g_{1}, \ldots, g_{N}\right\}$ stands for the linear span of $\left\{g_{1}, \ldots, g_{N}\right\}$. Moreover, for $\lambda \in\left[0, \frac{1}{2}\left[\right.\right.$ the operator $\left(I-\lambda K_{\mathbf{g}}\right)$ is invertible and for $N=1$ and $\left.\left.\lambda \in\right] \frac{1}{2}, 1\right]$ we have

$$
\operatorname{Ker}\left(I-\lambda K_{\mathbf{g}}\right)=\operatorname{LS}\left\{\mathbf{g}(\cdot)\left(\int_{0} \mathbf{g}^{2}(s) d s\right)^{\lambda-1}\right\} .
$$

On the other hand, the adjoint operator $K_{\mathbf{g}}^{*}$ for $K_{\mathbf{g}}$ is given by

$$
\left(K_{\mathbf{g}}^{*} \alpha\right)(t)=\mathbf{g}^{\tau}(t) \int_{t}^{1} G^{-1}(s) \mathbf{g}(s) \alpha(s) d s
$$

and satisfies

$$
\left\|K_{\mathbf{g}}^{*} \alpha\right\| \leqslant 2\|\alpha\| \quad \forall \alpha \in L^{2}([0,1]) .
$$

The above inequality follows from the fact that $\left\|K_{\mathbf{g}}^{*} \alpha\right\|^{2}=2\left\langle\alpha, K_{\mathbf{g}}^{*} \alpha\right\rangle$.

$\mathrm{R} \mathrm{e} \mathrm{m} \mathrm{a} \mathrm{r} \mathrm{k} \mathrm{1.} \mathrm{Inequalities} \mathrm{(5)} \mathrm{and} \mathrm{(9)} \mathrm{generalize} \mathrm{the} \mathrm{Hardy's} L^{2}$ inequality (3) which corresponds to the case when $\mathbf{g}(t)=1$ for all $t \in[0,1]$.

We will give some properties of the operators $K_{\mathbf{g}}$ and $K_{\mathbf{g}}^{*}$. 
First, $\left(I-K_{\mathbf{g}}^{*}\right)$ is an isometric operator on $L^{2}([0,1])$ and we have

$$
\left(I-K_{\mathbf{g}}\right)\left(I-K_{\mathbf{g}}^{*}\right)=I .
$$

By an easy calculation, we find

$$
\begin{aligned}
(\alpha, \beta) & =\left(\left(I-K_{\mathbf{g}}\right)\left(I-K_{\mathbf{g}}^{*}\right) \alpha, \beta\right) \\
& =(\alpha, \beta)-\left(K_{\mathbf{g}} \alpha, \beta\right)-\left(K_{\mathbf{g}}^{*} \alpha, \beta\right)+\left(K_{\mathbf{g}}^{*} \alpha, K_{\mathbf{g}}^{*} \beta\right),
\end{aligned}
$$

which implies

$$
\left(K_{\mathbf{g}} \alpha, \beta\right)+\left(K_{\mathbf{g}}^{*} \alpha, \beta\right)=\left(K_{\mathbf{g}}^{*} \alpha, K_{\mathbf{g}}^{*} \beta\right)=\left(\alpha, K_{\mathbf{g}}^{*} \beta\right)+\left(K_{\mathbf{g}}^{*} \alpha, \beta\right) .
$$

Now, we look to some functions $\mathbf{g}$ for which $\left(I-K_{\mathbf{g}}^{*}\right)$ take a simple form. Especially, in the case of $\mathbf{g}(t)=t^{a}, a>0$, we have

$$
\left(K_{\mathbf{g}}^{*} \mathbf{I}_{[0, t]}\right)(u)=\frac{2 a+1}{a}\left(1-\left(\frac{u}{t}\right)^{a}\right) \mathbf{I}_{[0, t]}(u) .
$$

Inequality (9) implies that $\left\|K_{\mathbf{g}}^{*}\right\| \leqslant 2$. So for $|\lambda|<\frac{1}{2}$ the operator $\left(I-\lambda K_{\mathbf{g}}^{*}\right)$ is invertible and its inverse can be expressed as follows:

$$
\left(I-\lambda K_{\mathbf{g}}^{*}\right)^{-1}=\sum_{n \geqslant 0} \lambda^{n}\left(K_{\mathbf{g}}^{*}\right)^{\circ n}
$$

It is not difficult to show that, for $\phi, \varphi \in L^{2}([0,1])$, we have

$$
\left\langle\left(I-\lambda K_{\mathbf{g}}^{*}\right) \phi,\left(I-\lambda K_{\mathbf{g}}^{*}\right) \varphi\right\rangle=\left\langle\left(I-(1-\lambda) K_{\mathbf{g}}^{*}\right) \phi,\left(I-(1-\lambda) K_{\mathbf{g}}^{*}\right) \varphi\right\rangle,
$$

which implies that

$$
\left\|\left(I-\lambda K_{\mathbf{g}}^{*}\right)\left(I-(1-\lambda) K_{\mathbf{g}}^{*}\right)^{-1} \alpha\right\|=\|\alpha\|,
$$

for any $\left.\lambda \in] \frac{1}{2}, 1\right]$ and $\alpha \in L^{2}([0,1])$. In other words, the operator (I$\left.\lambda K_{\mathbf{g}}^{*}\right)\left(I-(1-\lambda) K_{\mathbf{g}}^{*}\right)^{-1}$ is an isometric operator in $L^{2}([0,1])$.

3. A class of transformations of two Brownian motions. Let $(\Omega, \mathscr{F}, \mathbf{P})$ be a complete probability space and $\left(B_{t}\right)_{0 \leqslant t \leqslant 1}$ a standard Brownian motion with respect to its canonical filtration $\left(\mathscr{F}_{t}^{B}\right)_{0 \leqslant t \leqslant 1}$. Let $\left(\widetilde{B}_{t}\right)_{0 \leqslant t \leqslant 1}$ be another standard Brownian motion on the same probability space which is independent of $\left(B_{t}\right)_{0 \leqslant t \leqslant 1}$. Deheuvels [6] has shown that if $g$ is a measurable function such that

$$
\int_{0}^{t} \sqrt{u}|g(u)| d u<+\infty, \quad \text { for all } t \leqslant 1,
$$

then the process $\left(\tilde{X}_{t}\right)_{0 \leqslant t \leqslant 1}$ defined by $\tilde{X}_{t}=B_{t}+\int_{0}^{t} g(u) B_{u} d u$ is a Brownian motion if and only if $g(t)=0$ or $g(t)=-1 / t$. In this case the process $\tilde{X}_{t}$ takes the form

$$
\tilde{X}_{t}=B_{t}-\int_{0}^{t} \frac{B_{u}}{u} d u
$$


If we consider the transformation $T$ defined by $T(B)_{t}=B_{t}-\int_{0}^{t} u^{-1} B_{u} d u$ $(0 \leqslant t \leqslant 1)$, then we see that $T$ transforms the original Brownian motion into the new one. That is, $T$ leaves the Wiener measure invariant. We notice also that this transformation is closely related to the Hardy transform (2). A complete study of this transformation can be found in [11].

Recently, Wu and Yor [19] generalized this result to the case of two Brownian motions by considering the process $X$ given by

$$
X_{t}=B_{t}+\int_{0}^{t}\left(f(u) B_{u}+g(u) \widetilde{B}_{u}\right) d u
$$

where $f$ and $g$ are continuous and satisfy (12). They obtained that $X_{t}$ is a Brownian motion if and only if $f(t)= \pm \sqrt{\nu-\nu^{2}} / t$ and $g(t)=-\nu / t$ for some $\nu \in[0,1]$. In this case the process $X_{t}$ takes the form

$$
X_{t}=B_{t}-\int_{0}^{t}\left(\frac{\nu}{u} B_{u} \pm \frac{\sqrt{\nu-\nu^{2}}}{u} \widetilde{B}_{u}\right) d u .
$$

We note that the study of these processes has some applications in the context of financial equilibrium model (see [8]).

Taking into account Remark 1 , it seems interesting for us to study some similar questions to the above one by using the operator $\left(I-K_{\mathbf{g}}^{*}\right)$. Recall that this approach was used initially to construct some noncanonical representations of Brownian motion and then to evaluate the loss of the quantity of information for these representations. Before we continue our discussion, we note that the Gaussian structure facilitates the proofs of our results. On the other hand, it enables us to show that some properties may be deduced easily from a simple computation of the covariance function. Before stating the principal result of this section, denote

$$
X_{t}^{\lambda}:=\int\left(I-\lambda K_{\mathbf{g}}^{*}\right) \mathbf{I}_{[0, t]}(u) d B_{u},
$$

where $\lambda \in \mathbf{R}$ and $0 \leqslant t \leqslant 1$.

R e m a r k 2. It follows from (11) that, for $\left.\lambda \in] \frac{1}{2}, 1\right]$, the process

$$
B_{t}^{\lambda}=\int\left(I-\lambda K_{\mathbf{g}}^{*}\right)\left(I-(1-\lambda) K_{\mathbf{g}}^{*}\right)^{-1} \mathbf{I}_{[0, t]}(u) d B_{u}
$$

is a Brownian motion with respect to its own filtration and

$$
H_{t}(B)=H_{t}\left(B^{\lambda}\right) \oplus \operatorname{LS}\left\{\int_{0}^{t} \mathbf{g}(s)\left(\int_{0}^{s} \mathbf{g}^{2}(u) d u\right)^{\lambda-1} d B_{s}\right\} .
$$

In other words, the representation (14) of $B^{\lambda}$ is noncanonical with respect to $B$. 
Theorem 1. (a) For any $\lambda \in \mathbf{R}$,

$$
\left\{X_{t}^{\lambda} ; 0 \leqslant t \leqslant 1\right\} \stackrel{\text { law }}{=}\left\{X_{t}^{1-\lambda} ; 0 \leqslant t \leqslant 1\right\} .
$$

(b) Representation (13) of $X^{\lambda}$ is canonical with respect to $B$ if and only if $\lambda \leqslant \frac{1}{2}$. follows:

(c) For $\left.\lambda \in] \frac{1}{2}, 1\right], X^{\lambda}$ is canonically represented with respect to $B^{\lambda}$ as

$$
X_{t}^{\lambda}=\int\left(I-(1-\lambda) K_{\mathbf{g}}^{*}\right) \mathbf{I}_{[0, t]}(u) d B_{u}^{\lambda} .
$$

(d) For any $\lambda \notin[0,1]$,

$$
\left\{X_{t}^{\lambda} ; 0 \leqslant t \leqslant 1\right\} \stackrel{\text { law }}{=}\left\{B_{t} \pm \sqrt{\lambda^{2}-\lambda} \int K_{\mathbf{g}}^{*} \mathbf{I}_{[0, t]}(u) d \widetilde{B}_{u} ; 0 \leqslant t \leqslant 1\right\} .
$$

(e) For any $\lambda \in[0,1]$, the process

$$
X_{t}^{ \pm}:=\int\left(I-\lambda K_{\mathbf{g}}^{*}\right) \mathbf{I}_{[0, t]}(u) d B_{u} \pm \int \sqrt{\lambda-\lambda^{2}} K_{\mathbf{g}}^{*} \mathbf{I}_{[0, t]}(u) d \widetilde{B}_{u}
$$

are Brownian motions.

P r o o f. All processes are Gaussian, therefore in order to show (a)-(e) it is enough to prove that the covariances on both sides are equal.

The proof of (a) and (b) can be found in [13].

(c) Since for $\left.\lambda \in] \frac{1}{2}, 1\right]$ we have $(1-\lambda)<\frac{1}{2}$, assertion (c) follows from the definition of $B^{\lambda}$ and assertion (b).

(d) Using (10) the covariance of

$$
X_{t}^{\lambda}=\int\left(I-\lambda K_{\mathbf{g}}^{*}\right) \mathbf{I}_{[0, t]}(u) d B_{u}
$$

is given by

$$
\begin{aligned}
\mathbf{E}\left(X_{t}^{\lambda} X_{s}^{\lambda}\right)= & \int\left(I-\lambda K_{\mathbf{g}}^{*}\right) \mathbf{I}_{[0, t]}(u)\left(I-\lambda K_{\mathbf{g}}^{*}\right) \mathbf{I}_{[0, s]}(u) d u \\
= & t \wedge s-\lambda\left(K_{\mathbf{g}}^{*} \mathbf{I}_{[0, t]}, \mathbf{I}_{[0, s]}\right)-\lambda\left(K_{\mathbf{g}}^{*} \mathbf{I}_{[0, s]}, \mathbf{I}_{[0, t]}\right) \\
& +\lambda^{2}\left(K_{\mathbf{g}}^{*} \mathbf{I}_{[0, t]}, K_{\mathbf{g}}^{*} \mathbf{I}_{[0, s]}\right) \\
= & t \wedge s-\lambda\left(K_{\mathbf{g}}^{*} \mathbf{I}_{[0, t]}, K_{\mathbf{g}}^{*} \mathbf{I}_{[0, s]}\right)+\lambda^{2}\left(K_{\mathbf{g}}^{*} \mathbf{I}_{[0, t]}, K_{\mathbf{g}}^{*} \mathbf{I}_{[0, s]}\right) \\
= & t \wedge s+\left(\lambda^{2}-\lambda\right)\left(K_{\mathbf{g}}^{*} \mathbf{I}_{[0, t]}, K_{\mathbf{g}}^{*} \mathbf{I}_{[0, s]}\right)
\end{aligned}
$$

On the other hand, the covariance of $\tilde{X}_{t}^{\lambda}=B_{t} \pm \sqrt{\lambda-\lambda^{2}} \int K_{\mathbf{g}}^{*} \mathbf{I}_{[0, t]}(u) d \widetilde{B}_{u}$ is equal to

$$
\mathbf{E}\left(\widetilde{X}_{t}^{\lambda} \widetilde{X}_{s}^{\lambda}\right)=t \wedge s+\left(\lambda^{2}-\lambda\right)\left(K_{\mathbf{g}}^{*} \mathbf{I}_{[0, t]}, K_{\mathbf{g}}^{*} \mathbf{I}_{[0, s]}\right)
$$


(e) The covariance of $X_{t}^{ \pm}$is equal to

$$
\begin{aligned}
\mathbf{E}\left(X_{t}^{ \pm} X_{s}^{ \pm}\right)= & \mathbf{E}\left(X_{t}^{\lambda} X_{s}^{\lambda}\right)+\left(\lambda-\lambda^{2}\right)\left(K_{\mathbf{g}}^{*} \mathbf{I}_{[0, t]}, K_{\mathbf{g}}^{*} \mathbf{I}_{[0, s]}\right) \\
= & t \wedge s+\left(\lambda^{2}-\lambda\right)\left(K_{\mathbf{g}}^{*} \mathbf{I}_{[0, t]}, K_{\mathbf{g}}^{*} \mathbf{I}_{[0, s]}\right) \\
& +\left(\lambda-\lambda^{2}\right)\left(K_{\mathbf{g}}^{*} \mathbf{I}_{[0, t]}, K_{\mathbf{g}}^{*} \mathbf{I}_{[0, s]}\right)=t \wedge s,
\end{aligned}
$$

which implies that the processes $X^{ \pm}$are Brownian motions.

Theorem 1 is proved.

Now, if we exchange the roles of $B$ and $\widetilde{B}$ in (15) we can easily check that the resulting processes

$$
\widetilde{X}_{t}^{ \pm}:=\int\left(I-\lambda K_{\mathbf{g}}^{*}\right) \mathbf{I}_{[0, t]}(u) d \widetilde{B}_{u} \mp \int \sqrt{\lambda-\lambda^{2}} K_{\mathbf{g}}^{*} \mathbf{I}_{[0, t]}(u) d B_{u}
$$

are Brownian motions.

A natural question arises: Are they independent of $X^{ \pm}$? To answer this question it is enough to calculate

$$
\begin{aligned}
\mathbf{E}\left(X_{t}^{+} \tilde{X}_{s}^{+}\right)= & \sqrt{\lambda-\lambda^{2}}\left\{\left\langle\left(I-\lambda K_{\mathbf{g}}^{*}\right) \mathbf{I}_{[0, t]}, K_{\mathbf{g}}^{*} \mathbf{I}_{[0, s]}\right\rangle\right. \\
& \left.-\left\langle\left(I-\lambda K_{\mathbf{g}}^{*}\right) \mathbf{I}_{[0, s]}, K_{\mathbf{g}}^{*} \mathbf{I}_{[0, t]}\right\rangle\right\} \\
= & \sqrt{\lambda-\lambda^{2}}\left\{\left\langle\mathbf{I}_{[0, t]}, K_{\mathbf{g}}^{*} \mathbf{I}_{[0, s]}\right\rangle-\left\langle\mathbf{I}_{[0, s]}, K_{g}^{*} \mathbf{I}_{[0, t]}\right\rangle\right\} \\
= & \sqrt{\lambda-\lambda^{2}}\left\langle\mathbf{I}_{[0, s]},\left(K_{\mathbf{g}}-K_{\mathbf{g}}^{*}\right) \mathbf{I}_{[0, t]}\right\rangle .
\end{aligned}
$$

We conclude then that for any given $t$ the variables $X_{t}^{ \pm}$and $\tilde{X}_{t}^{ \pm}$are independent. But the processes $X^{ \pm}$and $\tilde{X}^{ \pm}$are not independent; hence we look for other Brownian motions which might be independent of $X$. This is given in the following proposition.

Proposition 1. For any $\lambda \in[0,1]$, the processes $Y^{ \pm}$defined by

$$
Y_{t}^{ \pm}:=\int\left(I-(1-\lambda) K_{\mathbf{g}}^{*}\right) \mathbf{I}_{[0, t]}(u) d \widetilde{B}_{u} \pm \int \sqrt{\lambda-\lambda^{2}} K_{\mathbf{g}}^{*} \mathbf{I}_{[0, t]}(u) d B_{u}
$$

are Brownian motions; each of them is independent of the corresponding process $\left(X^{ \pm}\right)$as defined in (15).

P r o o f. First notice that $\sqrt{(1-\lambda)-(1-\lambda)^{2}}=\sqrt{\lambda-\lambda^{2}}$. On the other hand, by changing $B$ and $\widetilde{B}$ in (15) we can see that the processes $Y_{t}^{ \pm}$ have the same form as $X_{t}^{ \pm}$. So, we obtain that the resulting processes are Brownian motions. That is, the processes $Y^{ \pm}$are Brownian motions. We have

$$
\begin{aligned}
\mathbf{E}\left(X_{t}^{+} Y_{s}^{+}\right)=\sqrt{\lambda-\lambda^{2}} & \left\{\left(I-(1-\lambda) K_{\mathbf{g}}^{*}\right) \mathbf{I}_{[0, s]}, K_{\mathbf{g}}^{*} \mathbf{I}_{[0, t]}\right\rangle \\
& \left.+\left\langle\left(I-\lambda K_{\mathbf{g}}^{*}\right) \mathbf{I}_{[0, t]}, K_{\mathbf{g}}^{*} \mathbf{I}_{[0, s]}\right\rangle\right\} \\
=\sqrt{\lambda-\lambda^{2}} & \left\{\left\langle\mathbf{I}_{[0, s]}, K_{\mathbf{g}}^{*} \mathbf{I}_{[0, t]}\right\rangle-\left\langle K_{\mathbf{g}}^{*} \mathbf{I}_{[0, s]}, K_{\mathbf{g}}^{*} \mathbf{I}_{[0, t]}\right\rangle\right. \\
& \left.+\left\langle\mathbf{I}_{[0, t]}, K_{\mathbf{g}}^{*} \mathbf{I}_{[0, s]}\right\rangle\right\}=0,
\end{aligned}
$$


where the last equality follows from (10). Moreover, $\mathbf{E}\left(X_{t}^{+} Y_{s}^{+}\right)=$ $\mathbf{E}\left(X_{s}^{+} Y_{t}^{+}\right)=0$, for all $s \leqslant t$, and the same for $X^{-}$and $Y^{-}$which complete the proof.

Now, we discuss some questions about the natural filtrations of $X^{ \pm}$, $Y^{ \pm}, B$, and $\widetilde{B}$. It is well known (see [5]) that if

$$
H_{t}\left(X^{ \pm}\right)=H_{t}(B) \oplus H_{t}(\widetilde{B}), \quad t \in(0,1),
$$

then the expansion (15) is the canonical representation or Cramér representation of the process $X^{ \pm}$. A necessary and sufficient condition is given in the following lemma.

Lemma 1. Representation (15) is canonical if and only if the family

$$
\left\{\left(I-\lambda K_{\mathbf{g}}^{*}\right) \mathbf{I}_{[0, t]}(u), \sqrt{\lambda-\lambda^{2}} K_{\mathbf{g}}^{*} \mathbf{I}_{[0, t]}(u)\right\}
$$

is complete in $L^{2}([0, t])$.

For a more general version of this result the reader can consult Lemma 3.1 in [5].

Proposition 2. (i) For any $\lambda \in[0,1]$ and $t \geqslant 0$, the filtration generated by $X^{ \pm}$and $Y^{ \pm}$is strictly smaller than that generated by $B$ and $\widetilde{B}$, i.e.,

$$
\mathscr{F}_{t}^{X^{ \pm}} \oplus \mathscr{F}_{t}^{Y^{ \pm}} \varsubsetneqq \mathscr{F}_{t}^{B} \oplus \mathscr{F}_{t}^{\widetilde{B}}
$$

Moreover, we have

$$
\begin{aligned}
& H_{t}(B, \widetilde{B})=H_{t}\left(X^{+}, Y^{+}\right) \oplus \operatorname{LS}\left\{\int_{0}^{t} \mathbf{g}(s) d\left(\sqrt{\lambda} B_{s}-\sqrt{1-\lambda} \widetilde{B}_{s}\right)\right\} \\
& H_{t}(B, \widetilde{B})=H_{t}\left(X^{-}, Y^{-}\right) \oplus \operatorname{LS}\left\{\int_{0}^{t} \mathbf{g}(s) d\left(\sqrt{\lambda} B_{s}+\sqrt{1-\lambda} \widetilde{B}_{s}\right)\right\} .
\end{aligned}
$$

(ii) For any $\lambda \in[0,1]$ and $t \geqslant 0$,

$$
\mathscr{F}_{t}^{X^{ \pm} \pm \sqrt{\lambda /(1-\lambda)} Y^{ \pm}}=\mathscr{F}_{t}^{B \pm \sqrt{\lambda /(1-\lambda)} \widetilde{B}} .
$$

P r o o f. (i) Since any element of $H_{t}(B, \widetilde{B})$ is a linear combination of $B_{s}, \widetilde{B}_{s}, s \leqslant t$, or its limit, it is sufficient to prove that for $\mathbf{f}, \mathbf{h} \in L^{2}[0,1]$ the expectations

$$
\mathbf{E}\left(X_{s}^{ \pm}\left(\int_{0}^{t} \mathbf{f}(u) d B_{u}+\int_{0}^{t} \mathbf{h}(u) d \widetilde{B}_{u}\right)\right)
$$

and

$$
\mathbf{E}\left(Y_{s}^{ \pm}\left(\int_{0}^{t} \mathbf{f}(u) d B_{u}+\int_{0}^{t} \mathbf{h}(u) d \widetilde{B}_{u}\right)\right)
$$

are equal to 0 for all $s \leqslant t$ if and only if $\mathbf{h}=\mp \sqrt{(1-\lambda) / \lambda} \mathbf{f}$ and $\mathbf{f} \in$ LS $\{\mathbf{g}\}$. That is, the variable $\left(\int_{0}^{t} \mathbf{g}(s) d\left(\sqrt{\lambda} B_{s}-\sqrt{1-\lambda} \widetilde{B}_{s}\right)\right)$ (respectively, 
$\left.\int_{0}^{t} \mathbf{g}(s) d\left(\sqrt{\lambda} B_{s}+\sqrt{1-\lambda} \widetilde{B}_{s}\right)\right)$ is independent of $\mathscr{F}_{t}^{X^{+}} \oplus \mathscr{F}_{t}^{Y^{+}}$(respectively, $\left.\mathscr{F}_{t}^{X^{-}} \oplus \mathscr{F}_{t}^{Y^{-}}\right)$.

(ii) Follows directly from the relation

$$
X_{t}^{ \pm} \pm \sqrt{\frac{\lambda}{1-\lambda}} Y_{t}=B_{t} \pm \sqrt{\frac{\lambda}{1-\lambda}} \widetilde{B}_{t}, \quad \text { for all } t \geqslant 0 .
$$

This completes the proof.

$\mathrm{R}$ e $\mathrm{m}$ a r k 3. Let $\boldsymbol{\alpha}=(\sqrt{\lambda}, \mp \sqrt{1-\lambda})$. Then the (two-dimensional) Brownian motion $\left(X^{ \pm}, Y^{ \pm}\right)$given by

$$
\begin{aligned}
& X_{t}^{ \pm}=B_{t}-\left(\int \lambda K_{\mathbf{g}}^{*} \mathbf{I}_{[0, t]}(u) d B_{u} \mp \int \sqrt{\lambda-\lambda^{2}} K_{\mathbf{g}}^{*} \mathbf{I}_{[0, t]}(u) d \widetilde{B}_{u}\right), \\
& Y_{t}^{ \pm}=\widetilde{B}_{t}-\left(\mp \int \sqrt{\lambda-\lambda^{2}} K_{\mathbf{g}}^{*} \mathbf{I}_{[0, t]}(u) d B_{u}+\int(1-\lambda) K_{\mathbf{g}}^{*} \mathbf{I}_{[0, t]}(u) d \widetilde{B}_{u}\right)
\end{aligned}
$$

can be rewritten as

$$
\left(X_{t}^{ \pm}, Y_{t}^{ \pm}\right)^{\tau}=\left(B_{t}, \widetilde{B}_{t}\right)^{\tau}-\boldsymbol{\alpha}^{\tau} \boldsymbol{\alpha} \int K_{\mathbf{g}}^{*} \mathbf{I}_{[0, t]}(u) d\left(B_{u}, \widetilde{B}_{u}\right)^{\tau} .
$$

It seems natural to us to extend the result of Proposition 2 to the $n$-dimensional case in the following way. Let $\left\{\mathbf{B}_{t}=\left(B_{t}^{1}, \ldots, B_{t}^{n}\right), t \geqslant 0\right\}$ be an $n$-dimensional Brownian motion and let $\boldsymbol{\alpha}=\left(\alpha_{1}, \ldots, \alpha_{n}\right)$ be such that $\sum_{i=1}^{n} \alpha_{i}^{2}=1$. Then the process

$$
\mathbf{X}_{t}=\mathbf{B}_{t}-\boldsymbol{\alpha}^{\tau} \boldsymbol{\alpha} \int K_{\mathbf{g}}^{*} \mathbf{I}_{[0, t]}(u) d \mathbf{B}_{u}
$$

is an $n$-dimensional Brownian motion which satisfies

$$
H_{t}(\mathbf{B})=H_{t}(\mathbf{X}) \oplus \operatorname{LS}\left\{\sum_{i=1}^{n} \alpha_{i} \int_{0}^{t} \mathbf{g}(s) d B_{s}^{i}\right\} .
$$

This result can be seen as an extension of Theorem 2.2 in [19] and a particular case of [15].

4. An orthogonal decomposition of Brownian filtrations. We start by pointing out an orthogonal decomposition of the Brownian filtration due to Hibino, Hitsuda, and Muraoka [14]. Let $\left(B_{t}\right)_{t \geqslant 0}$ be a standard Brownian motion and define the process

$$
B_{t}^{\mathbf{g}}:=\int_{0}^{t}\left(I-K_{\mathbf{g}}^{*}\right) \mathbf{I}_{[0, t]}(u) d B_{u} .
$$

In [14] it has been shown that the canonical filtration $\left(\mathscr{F}_{t}^{B}\right)$ of $\left(B_{t}\right)_{t \geqslant 0}$ can be decomposed into the direct sum of two independents $\sigma$-algebras:

$$
\mathscr{F}_{t}^{B}=\mathscr{G}_{t} \oplus \sigma\left(\int_{0}^{t} \mathbf{g}(u) d B_{u}\right)
$$


for all $t \geqslant 0$, where the $\sigma$-algebra $\mathscr{G}_{t}$ is given by $\mathscr{G}_{t}:=\sigma\left(B_{s}^{\mathrm{g}} ; s \leqslant t\right)$. Using the Gaussian structure of $\left(B_{t}\right)_{t \geqslant 0}$ we can see that (18) is equivalent to

$$
H_{t}(B)=H_{t}\left(B^{\mathbf{g}}\right) \oplus \operatorname{LS}\left\{\int_{0}^{t} \mathbf{g}(u) d B_{u}\right\}
$$

where $H_{t}(B)$ (respectively, $H_{t}\left(B^{\mathbf{g}}\right)$ ) is the Gaussian space associated with $B$ (respectively, $B^{\mathbf{g}}$ ) and $\operatorname{LS}\left\{\int_{0}^{t} \mathbf{g}(u) d B_{u}\right\}$ means the linear span of $\left\{\int_{0}^{t} \mathbf{g}(u) d B_{u}\right\}$.

Iterating this procedure, one can construct a new family of Brownian motions $B_{t}^{N, \mathbf{g}}$ defined by

$$
B_{t}^{N, \mathbf{g}}:=\int_{0}^{t}\left(I-K_{\mathbf{g}}^{*}\right)^{N} \mathbf{I}_{[0, t]}(u) d B_{u}
$$

and get an orthogonal decomposition of $\mathscr{F}_{t}^{B}$ and $H_{t}(B)$ as follows:

$$
\begin{aligned}
\mathscr{F}_{t}^{B} & =\mathscr{F}_{t}^{B^{N, \mathbf{g}}} \oplus \bigoplus_{k=1}^{N} \sigma\left(\int_{0}^{t}\left(I-K_{\mathbf{g}}^{*}\right)^{k-1} \mathbf{g}(u) d B_{u}\right), \\
H_{t}(B) & =H_{t}\left(B^{N, \mathbf{g}}\right) \oplus \bigoplus_{k=1}^{N} \operatorname{LS}\left\{\int_{0}^{t}\left(I-K_{\mathbf{g}}^{*}\right)^{k-1} \mathbf{g}(u) d B_{u}\right\} .
\end{aligned}
$$

$\mathrm{R}$ e mark 4. The fact that $\left\{\left(I-K_{\mathbf{g}}^{*}\right)^{k} \mathbf{g} ; k \in \mathbf{N}\right\}$ corresponds to the orthogonalization of $\left\{\varphi_{k}(s)=\mathbf{g}(s)\left(\ln \int_{0}^{s} \mathbf{g}^{2}(u) d u\right)^{k-1} ; k \in \mathbf{N}\right\}$ which is complete in $L^{2}([0, t])$ leads to conclude that

$$
\begin{aligned}
\mathscr{F}_{t}^{B} & =\bigoplus_{k=1}^{\infty} \sigma\left(\int_{0}^{t}\left(I-K_{\mathbf{g}}^{*}\right)^{k-1} \mathbf{g}(u) d B_{u}\right)=\bigoplus_{k=1}^{\infty} \sigma\left(\int_{0}^{t} \mathbf{g}(u) d B_{u}^{k-1, \mathbf{g}}\right), \\
H_{t}(B) & =\bigoplus_{k=1}^{\infty} \operatorname{LS}\left\{\int_{0}^{t}\left(I-K_{\mathbf{g}}^{*}\right)^{k-1} \mathbf{g}(u) d B_{u}\right\}=\bigoplus_{k=1}^{\infty} \operatorname{LS}\left\{\int_{0}^{t} \mathbf{g}(u) d B_{u}^{k-1, \mathbf{g}}\right\} .
\end{aligned}
$$

In term of filtrations, this is due to the fact that $\mathscr{F}_{t}^{B^{N, 8}}$ decreases as $N \rightarrow \infty$ to the trivial $\sigma$-field. On the other hand, we conclude that for fixed $t \geqslant 0$, the sequence $\left(\int_{0}^{t} \mathbf{g}(u) d B_{u}^{N, \mathbf{g}}\right)_{N \geqslant 0}$ is not strongly $L^{2}$-convergent, but converges weakly to 0 in $L^{2}$. For more details on the subject the reader can consult [11], [12], and [19].

4.1. A first construction of orthogonal decompositions of Brownian filtrations. We start by establishing the connection with a result of [19] on construction of orthogonal decomposition of Brownian filtrations. Let $\left(B_{t}\right)$ and $\left(\widetilde{B}_{t}\right)$ be two independent Brownian motions. Consider the function $\mathbf{g}(t)=t^{a}, t \in[0,1]$, and $\lambda=a /(2 a+1)$, where $a>0$. In this case the processes defined by (15) can be written as follows:

$$
X_{t}^{ \pm}=\int_{0}^{t}\left(\frac{u}{t}\right)^{a} d B_{u} \pm \int_{0}^{t} \sqrt{\frac{a+1}{a}}\left(1-\left(\frac{u}{t}\right)^{a}\right) d \widetilde{B}_{u}
$$


and are Brownian motions.

Let $c= \pm \sqrt{a /(a+1)}$, then $0 \leqslant|c|<1$ and $a=c^{2} /\left(1-c^{2}\right)$. In this case the processes $X_{t}^{ \pm}$take the form

$$
X_{t}^{ \pm}=\int_{0}^{t}\left(\frac{u}{t}\right)^{a} d B_{u}+\frac{1}{c} \int_{0}^{t}\left(1-\left(\frac{u}{t}\right)^{a}\right) d \widetilde{B}_{u}
$$

and satisfy the stochastic differential equation

$$
d X_{t}^{ \pm}=d B_{t}+\frac{c \widetilde{B}_{t}-c^{2} X_{t}^{ \pm}}{\left(1-c^{2}\right) t} d t
$$

It follows from Proposition 1 that the corresponding processes

$$
Y_{t}^{ \pm}=\frac{1}{c} \int_{0}^{t}\left(1-\left(\frac{u}{t}\right)^{a}\right) d B_{u}-\int_{0}^{t}\left(\frac{1}{a}-\frac{a+1}{a}\left(\frac{u}{t}\right)^{a}\right) d \widetilde{B}_{u}
$$

are Brownian motions; each of them is independent of $X^{ \pm}$. Moreover, they satisfy the following stochastic differential equation:

$$
d Y_{t}^{ \pm}=d \widetilde{B}_{t}+\frac{c B_{t}-\left(1-c^{2}\right) \widetilde{B}_{t}-c^{2} Y_{t}^{ \pm}}{\left(1-c^{2}\right) t} d t .
$$

We point out that these processes as well as their filtrations were well studied by $\mathrm{Wu}$ and Yor in [19]. Iterating this procedure, since $T$ transforms two independent Brownian motions into two ones, Wu and Yor define two sequences of stochastic processes $X^{ \pm, n+1}$ and $Y^{ \pm, n+1}$ which are independent of each other. This allows them to get a new orthogonal decomposition of Brownian filtrations.

It is well known that if $\left(B_{t}\right)_{t \geqslant 0}$ is a Brownian motion, then the process $\left(B_{t}^{\mathbf{g}}\right)_{t \geqslant 0}$ defined in (17) is a Brownian motion and its natural filtration $\left(\mathscr{F}_{t}^{B^{\mathbf{B}}}\right)_{t \geqslant 0}$ is strictly smaller than $\left(\mathscr{F}_{t}^{B}\right)_{t \geqslant 0}$. On the other hand, since $\left(B_{t}\right)_{t \geqslant 0}$ and $\left(\widetilde{B}_{t}\right)_{t \geqslant 0}$ are independent, the processes $\left(B_{t}^{\mathbf{g}}\right)_{t \geqslant 0}$ and $\left(\widetilde{B_{t}^{\mathbf{g}}}\right)_{t \geqslant 0}$ are also independent. Using the same argument as in Theorem 1 and Proposition 1 we know that the processes

$$
\begin{aligned}
X_{t}^{ \pm, \mathbf{g}}= & \int\left(I-\lambda K_{\mathbf{g}}^{*}\right) \mathbf{I}_{[0, t]}(u) d B_{u}^{\mathbf{g}} \pm \int \sqrt{\lambda-\lambda^{2}} K_{\mathbf{g}}^{*} \mathbf{I}_{[0, t]}(u) d \widetilde{B_{u}^{\mathbf{g}}} \\
= & \int\left(I-K_{\mathbf{g}}^{*}\right)\left(I-\lambda K_{\mathbf{g}}^{*}\right) \mathbf{I}_{[0, t]}(u) d B_{u} \\
& \pm \int \sqrt{\lambda-\lambda^{2}}\left(I-K_{\mathbf{g}}^{*}\right) K_{\mathbf{g}}^{*} \mathbf{I}_{[0, t]}(u) d \widetilde{B_{u}} \\
= & \int\left(I-K_{\mathbf{g}}^{*}\right) \mathbf{I}_{[0, t]}(u) d X_{u}^{ \pm}
\end{aligned}
$$

are Brownian motions independent of $Y^{ \pm, \mathbf{g}}$ given by

$$
\begin{aligned}
Y_{t}^{ \pm, \mathbf{g}} & =\int\left(I-(1-\lambda) K_{\mathbf{g}}^{*}\right) \mathbf{I}_{[0, t]}(u) d \widetilde{B_{u}^{\mathbf{g}}} \pm \int \sqrt{\lambda-\lambda^{2}} K_{\mathbf{g}}^{*} \mathbf{I}_{[0, t]}(u) d B_{u}^{\mathbf{g}} \\
& =\int\left(I-K_{\mathbf{g}}^{*}\right) \mathbf{I}_{[0, t]}(u) d Y_{u}^{ \pm} .
\end{aligned}
$$


We know that the $\sigma$-algebras generated by $B^{\mathrm{g}}$ and $\widetilde{B^{\mathbf{g}}}$ are strictly smaller than those generated by $B$ and $\widetilde{B}$, respectively. Furthermore, for all $s \leqslant t$, we have

$$
\mathbf{E}\left(X_{s}^{ \pm, \mathbf{g}} \int_{0}^{t} \mathbf{g}(u) d X_{u}^{ \pm}\right)=0
$$

which implies that the variable $\int_{0}^{t} \mathbf{g}(u) d X_{u}^{ \pm}$is independent of $\mathscr{F}_{t}^{X^{ \pm, \mathbf{g}}}$. So, we have $\mathscr{F}_{t}^{X^{ \pm, \mathbf{g}}} \subsetneq \mathscr{F}_{t}^{X^{ \pm}}$.

Iterating this procedure, we define

$$
\begin{aligned}
X_{t}^{ \pm, n, \mathbf{g}}:= & \int\left(I-\lambda K_{\mathbf{g}}^{*}\right) \mathbf{I}_{[0, t]}(u) d B_{u}^{n, \mathbf{g}} \\
& \pm \int \sqrt{\lambda-\lambda^{2}} K_{\mathbf{g}}^{*} \mathbf{I}_{[0, t]}(u) d \widetilde{B_{u}^{n, \mathbf{g}}} \\
Y_{t}^{ \pm, n, \mathbf{g}}:= & \int\left(I-(1-\lambda) K_{\mathbf{g}}^{*}\right) \mathbf{I}_{[0, t]}(u) d \widetilde{B_{u}^{n, \mathbf{g}}} \\
& \pm \int \sqrt{\lambda-\lambda^{2}} K_{\mathbf{g}}^{*} \mathbf{I}_{[0, t]}(u) d B_{u}^{n, \mathbf{g}}
\end{aligned}
$$

Thanks to the representations

$$
\begin{aligned}
& {B_{t}^{n, \mathbf{g}}}^{=}=\int_{0}^{t}\left(I-K_{\mathbf{g}}^{*}\right)^{n} \mathbf{I}_{[0, t]}(u) d B_{u}, \\
& \widetilde{B_{t}^{n, \mathbf{g}}}=\int_{0}^{t}\left(I-K_{\mathbf{g}}^{*}\right)^{n} \mathbf{I}_{[0, t]}(u) d \widetilde{B}_{u}
\end{aligned}
$$

we have

$$
\begin{aligned}
& B_{t}^{n+1, \mathbf{g}}=\int_{0}^{t}\left(I-K_{\mathbf{g}}^{*}\right) \mathbf{I}_{[0, t]}(u) d B_{u}^{n, \mathbf{g}}, \\
& \widetilde{B_{t}^{n+1, \mathbf{g}}}=\int_{0}^{t}\left(I-K_{\mathbf{g}}^{*}\right) \mathbf{I}_{[0, t]}(u) d \widetilde{B_{u}^{n, \mathbf{g}}} .
\end{aligned}
$$

As a consequence of the above representations we have the following proposition.

Proposition 3. (a) For $n \geqslant 0$ and $t \geqslant 0$,

$$
\begin{aligned}
& X_{t}^{ \pm, n+1, \mathbf{g}}=\int\left(I-K_{\mathbf{g}}^{*}\right) \mathbf{I}_{[0, t]}(u) d X_{u}^{ \pm, n, \mathbf{g}}, \\
& Y_{t}^{ \pm, n+1, \mathbf{g}}=\int\left(I-K_{\mathbf{g}}^{*}\right) \mathbf{I}_{[0, t]}(u) d Y_{u}^{ \pm, n, \mathbf{g}} .
\end{aligned}
$$

(b) For $\mathbf{f}, \mathbf{h} \in L^{2}[0,1]$,

$$
\begin{aligned}
& \mathbf{E}\left(X_{t}^{ \pm, n+1, \mathbf{g}} \int_{0}^{1} \mathbf{f}(u) d X_{u}^{ \pm, n, \mathbf{g}}\right)=0 \\
& \mathbf{E}\left(Y_{t}^{ \pm, n+1, g} \int_{0}^{1} \mathbf{h}(u) d Y_{u}^{ \pm, n, \mathbf{g}}\right)=0
\end{aligned}
$$

for all $t \leqslant 1$ if and only if $\mathbf{f}, \mathbf{h} \in \operatorname{LS}\{\mathbf{g}\}$. 
P r o o f. Assertion (a) follows from (24), (25) and the definition of $X_{t}^{ \pm, n, \mathbf{g}}$ and $Y_{t}^{ \pm, n, \mathbf{g}}$.

(b) It follows from (26) that, for $t \leqslant 1$,

$$
\mathbf{E}\left(X_{t}^{ \pm, n+1, \mathbf{g}} \int_{0}^{1} \mathbf{f}(u) d X_{u}^{ \pm, n, \mathbf{g}}\right)=\int_{0}^{1} \mathbf{f}(u)\left(I-K_{\mathbf{g}}^{*}\right) \mathbf{I}_{[0, t]}(u) d u,
$$

and this implies $\int_{0}^{t} \mathbf{I}_{[0, t]}(u)\left(I-K_{\mathbf{g}}\right) \mathbf{f}(u) d u=0$ for $t \in[0,1]$. This is reduced to

$$
\left(I-K_{\mathbf{g}}\right) \mathbf{f}=0,
$$

that is, $\mathbf{f} \in \operatorname{Ker}\left(I-K_{\mathbf{g}}\right)$. So, (6) completes the proof.

For each $n \geqslant 0$ the processes $X^{ \pm, n+1, \mathbf{g}}$ and $Y^{ \pm, n+1, \mathrm{~g}}$ are Brownian motions and

$$
\begin{aligned}
& \mathscr{F}_{t}^{X^{ \pm}} \supsetneqq \mathscr{F}_{t}^{X^{ \pm, 1, \mathbf{g}}} \supsetneqq \cdots \supsetneqq \mathscr{F}_{t}^{X^{ \pm, n, \mathbf{g}}} \supsetneqq \cdots, \\
& \mathscr{F}_{t}^{Y^{ \pm}} \supsetneqq \mathscr{F}_{t}^{Y^{ \pm, 1, \mathbf{g}}} \supsetneqq \cdots \cdots \mathscr{F}_{t}^{Y^{ \pm, n,}} \supsetneqq \cdots \cdots
\end{aligned}
$$

Moreover, we obtain an orthogonal decomposition, similar to (20), of the filtrations generated by $X^{ \pm}$and $Y^{ \pm}$as follows:

$$
\mathscr{F}_{t}^{X^{ \pm}}=\bigoplus_{n=0}^{\infty} \sigma\left(\int_{0}^{t} \mathbf{g}(u) d X_{u}^{ \pm, n, \mathbf{g}}\right), \quad \text { and } \quad \mathscr{F}_{t}^{Y^{ \pm}}=\bigoplus_{n=0}^{\infty} \sigma\left(\int_{0}^{t} \mathbf{g}(u) d Y_{u}^{ \pm, n, \mathbf{g}}\right) .
$$

A natural question arises: Are representations (21) and (22) canonical or Cramér representations of the processes $X^{ \pm, n, \mathbf{g}}$ and $Y^{ \pm, n, \mathbf{g}}$ ? Note that the processes $\left(X^{ \pm, n, \mathbf{g}}, Y^{ \pm, n, \mathbf{g}}\right)$ and $\left(X^{ \pm}, Y^{ \pm}\right)$have the same form with $\left(B^{n, \mathbf{g}}, \widetilde{B^{n, \mathbf{g}}}\right)$ instead $(B, \widetilde{B})$. So, the answer of this question to the same as the one given in Proposition 2.

Proposition 4. (i) For any $\lambda \in[0,1]$ and all $n \geqslant 0$ representations (21) and (22) are not canonical. That is, for any $t \geqslant 0$

$$
\mathscr{F}_{t}^{X^{ \pm, n, \mathbf{g}}} \oplus \mathscr{F}_{t}^{Y^{ \pm, n, \mathbf{g}}} \varsubsetneqq \mathscr{F}_{t}^{B^{n, \mathbf{g}}} \oplus \mathscr{F}_{t}^{\widetilde{B^{n, \mathbf{g}}}}
$$

Moreover,

$$
\begin{aligned}
H_{t}\left(B^{n, \mathbf{g}}, \widetilde{B^{n, \mathbf{g}}}\right)= & H_{t}\left(X^{+, n, \mathbf{g}}, Y^{+, n, \mathbf{g}}\right) \\
& \oplus \operatorname{LS}\left\{\int_{0}^{t} \mathbf{g}(s) d\left(\sqrt{\lambda} B_{s}^{n, \mathbf{g}}-\sqrt{1-\lambda} \widetilde{B_{s}^{n, \mathbf{g}}}\right)\right\}, \\
H_{t}\left(B^{n, \mathbf{g}}, \widetilde{B^{n, \mathbf{g}}}\right)= & H_{t}\left(X^{-, n, \mathbf{g}}, Y^{-, n, \mathbf{g}}\right) \\
& \oplus \operatorname{LS}\left\{\int_{0}^{t} \mathbf{g}(s) d\left(\sqrt{\lambda} B_{s}^{n, \mathbf{g}}+\sqrt{1-\lambda} \widetilde{B_{s}^{n, \mathbf{g}}}\right)\right\} .
\end{aligned}
$$

(ii) For any $\lambda \in[0,1]$ and $t \geqslant 0$,

$$
\mathscr{F}_{t}^{X^{ \pm, n, \mathbf{g}} \pm \sqrt{\lambda /(1-\lambda)} Y^{ \pm, n, \mathbf{g}}}=\mathscr{F}_{t}^{B^{n, \mathbf{g}} \pm \sqrt{\lambda /(1-\lambda)} \widetilde{B^{n, \mathbf{g}}}}
$$


(iii) For all $0 \leqslant n<m$, the $\sigma$-algebra $\mathscr{F}_{t}^{X^{ \pm, n, \mathbf{g}}} \oplus \mathscr{F}_{t}^{Y^{ \pm, n, \mathbf{g}}}$ is not contained in $\mathscr{F}_{t}^{B^{m, \mathbf{g}}} \oplus \mathscr{F}_{t}^{B^{m, \mathbf{g}}}$.

P r o o f. Here we show only (iii) for $m=n+1$. For this it suffices to see that

$$
\mathbf{E}\left(X_{s}^{+, n, \mathbf{g}} \int_{0}^{t} \mathbf{g}(s) d B_{s}^{n, \mathbf{g}}\right)=\int_{0}^{t}\left(I-\lambda K_{\mathbf{g}}^{*}\right) \mathbf{g}(u)\left(I-K_{\mathbf{g}}^{*}\right) \mathbf{I}_{[0, t]}(u) d u \neq 0 .
$$

$\mathrm{R}$ e $\mathrm{m}$ a r $\mathrm{k}$ 5. Using representation (23) one can rewrite (27) as follows:

$$
\begin{aligned}
H_{t}\left(B^{n, \mathbf{g}}, \widetilde{B^{n, \mathbf{g}}}\right)= & H_{t}\left(X^{+, n, \mathbf{g}}, Y^{+, n, \mathbf{g}}\right) \\
& \oplus \operatorname{LS}\left\{\int_{0}^{t}\left(I-K_{\mathbf{g}}^{*}\right)^{n} \mathbf{g}(s) d\left(\sqrt{\lambda} B_{s}-\sqrt{1-\lambda} \widetilde{B}_{s}\right)\right\}, \\
H_{t}\left(B^{n, \mathbf{g}}, \widetilde{B^{n, \mathbf{g}}}\right)= & H_{t}\left(X^{-, n, \mathbf{g}}, Y^{-, n, \mathbf{g}}\right) \\
& \oplus \operatorname{LS}\left\{\int_{0}^{t}\left(I-K_{\mathbf{g}}^{*}\right)^{n} \mathbf{g}(s) d\left(\sqrt{\lambda} B_{s}+\sqrt{1-\lambda} \widetilde{B}_{s}\right)\right\} .
\end{aligned}
$$

R e m a r k 6. Since $\left(\int_{0}^{t} \mathbf{g}(u) d X_{u}^{ \pm, n, \mathbf{g}}\right)_{n \geqslant 1}$ is an orthonormal system in $L^{2}(\mathbf{P})$, we conclude that for fixed $t \geqslant 0$, the sequence $\left(\int_{0}^{t} \mathbf{g}(u) d X_{u}^{ \pm, n, \mathbf{g}}\right)_{n \geqslant 1}$ is not strongly $L^{2}$-convergent, but converges weakly to 0 in $L^{2}$.

4.2. A second construction of orthogonal decompositions of Brownian filtrations. In this section we will present other processes, leading to the same conclusion as that of the preceding section. Let $\left(B_{t}\right)$ and let $\left(\widetilde{B}_{t}\right)$ be two independent Brownian motions and $\left.\left.\lambda \in\right] \frac{1}{2}, 1\right]$. It is well known (see, for example, [13]) that if $\left(B_{t}\right)_{t \geqslant 0}$ is a Brownian motion, then the process $\left(B_{t}^{\lambda}\right)$ defined in (14) is a Brownian motion and its natural filtration $\left(\mathscr{F}_{t}^{B^{\lambda}}\right)$ is strictly smaller than $\left(\mathscr{F}_{t}^{B}\right)$. On the other hand, since $\left(B_{t}\right)_{t \geqslant 0}$ and $\left(\widetilde{B}_{t}\right)_{t \geqslant 0}$ are independent, the processes $\left(B_{t}^{\lambda}\right)_{t \geqslant 0}$ and $\left(\widetilde{B_{t}^{\lambda}}\right)_{t \geqslant 0}$ are also independent. Using the same argument as in Theorem 1 and Proposition 1 we deduce that the processes

$$
\begin{aligned}
X_{t}^{ \pm, \lambda}= & \int\left(I-\lambda K_{\mathbf{g}}^{*}\right) \mathbf{I}_{[0, t]}(u) d B_{u}^{\lambda} \pm \int \sqrt{\lambda-\lambda^{2}} K_{\mathbf{g}}^{*} \mathbf{I}_{[0, t]}(u) d \widetilde{B_{u}^{\lambda}} \\
= & \int\left(I-\lambda K_{\mathbf{g}}^{*}\right)\left(I-(1-\lambda) K_{\mathbf{g}}^{*}\right)^{-1}\left(I-\lambda K_{\mathbf{g}}^{*}\right) \mathbf{I}_{[0, t]}(u) d B_{u} \\
& \pm \int \sqrt{\lambda-\lambda^{2}}\left(I-\lambda K_{\mathbf{g}}^{*}\right)\left(I-(1-\lambda) K_{\mathbf{g}}^{*}\right)^{-1} K_{\mathbf{g}}^{*} \mathbf{I}_{[0, t]}(u) d \widetilde{B}_{u} \\
= & \int\left(I-\lambda K_{\mathbf{g}}^{*}\right)\left(I-(1-\lambda) K_{\mathbf{g}}^{*}\right)^{-1} \mathbf{I}_{[0, t]}(u) d X_{u}^{ \pm}
\end{aligned}
$$

are Brownian motions independent of $Y^{ \pm, \lambda}$ given by

$$
\begin{aligned}
Y_{t}^{ \pm, \lambda} & =\int\left(I-(1-\lambda) K_{\mathbf{g}}^{*}\right) \mathbf{I}_{[0, t]}(u) d \widetilde{B_{u}^{\lambda}} \pm \int \sqrt{\lambda-\lambda^{2}} K_{\mathbf{g}}^{*} \mathbf{I}_{[0, t]}(u) d B_{u}^{\lambda} \\
& =\int\left(I-\lambda K_{\mathbf{g}}^{*}\right)\left(I-(1-\lambda) K_{\mathbf{g}}^{*}\right)^{-1} \mathbf{I}_{[0, t]}(u) d Y_{u}^{ \pm} .
\end{aligned}
$$


Furthermore, for all $s \leqslant t$, we have

$$
\mathbf{E}\left(X_{s}^{ \pm, \lambda} \int_{0}^{t} \mathbf{g}(s)\left(\int_{0}^{s} \mathbf{g}^{2}(u) d u\right)^{\lambda-1} d X_{s}^{ \pm}\right)=0,
$$

which implies that the variable $\int_{0}^{t} \mathbf{g}(s)\left(\int_{0}^{s} \mathbf{g}^{2}(u) d u\right)^{\lambda-1} d X_{s}^{ \pm}$is independent of $\mathscr{F}_{t}^{X^{ \pm, \lambda}}$. So, we have $\mathscr{F}_{t}^{X^{ \pm, \lambda}} \subsetneq \mathscr{F}_{t}^{X^{ \pm}}$.

Now, we define two sequences of Brownian motions

$$
\begin{aligned}
X_{t}^{ \pm, n, \lambda}:= & \int\left(I-\lambda K_{\mathbf{g}}^{*}\right) \mathbf{I}_{[0, t]}(u) d B_{u}^{n, \lambda} \pm \int \sqrt{\lambda-\lambda^{2}} K_{\mathbf{g}}^{*} \mathbf{I}_{[0, t]}(u) d \widetilde{B_{u}^{n, \lambda}} \\
Y_{t}^{ \pm, n, \lambda}:= & \int\left(I-(1-\lambda) K_{\mathbf{g}}^{*}\right) \mathbf{I}_{[0, t]}(u) d \widetilde{B_{u}^{n, \lambda}} \\
& \pm \int \sqrt{\lambda-\lambda^{2}} K_{\mathbf{g}}^{*} \mathbf{I}_{[0, t]}(u) d B_{u}^{n, \lambda}
\end{aligned}
$$

Thanks to the representations

$$
\begin{aligned}
B_{t}^{n, \lambda} & =\int_{0}^{t}\left(\left(I-\lambda K_{\mathbf{g}}^{*}\right)\left(I-(1-\lambda) K_{\mathbf{g}}^{*}\right)^{-1}\right)^{n} \mathbf{I}_{[0, t]}(u) d B_{u}, \\
\widetilde{B_{t}^{n, \lambda}} & =\int_{0}^{t}\left(\left(I-\lambda K_{\mathbf{g}}^{*}\right)\left(I-(1-\lambda) K_{\mathbf{g}}^{*}\right)^{-1}\right)^{n} \mathbf{I}_{[0, t]}(u) d \widetilde{B}_{u},
\end{aligned}
$$

we have the following proposition.

Proposition 5. (a) For $n \geqslant 0$ and $t \geqslant 0$,

$$
\begin{aligned}
X_{t}^{ \pm, n+1, \lambda} & =\int\left(\left(I-\lambda K_{\mathbf{g}}^{*}\right)\left(I-(1-\lambda) K_{\mathbf{g}}^{*}\right)^{-1}\right) \mathbf{I}_{[0, t]}(u) d X_{u}^{ \pm, n, \lambda}, \\
Y_{t}^{ \pm, n+1, \lambda} & =\int\left(\left(I-\lambda K_{\mathbf{g}}^{*}\right)\left(I-(1-\lambda) K_{\mathbf{g}}^{*}\right)^{-1}\right) \mathbf{I}_{[0, t]}(u) d Y_{u}^{ \pm, n, \lambda} .
\end{aligned}
$$

(b) For $\mathbf{f}, \mathbf{h} \in L^{2}[0,1]$,

$$
\begin{aligned}
& \mathbf{E}\left(X_{t}^{ \pm, n+1, \lambda} \int_{0}^{1} \mathbf{f}(u) d X_{u}^{ \pm, n, \lambda}\right)=0 \\
& \mathbf{E}\left(Y_{t}^{ \pm, n+1, \lambda} \int_{0}^{1} \mathbf{h}(u) d Y_{u}^{ \pm, n, \lambda}\right)=0
\end{aligned}
$$

for all $t \leqslant 1$ if and only if $\mathbf{f}, \mathbf{h} \in \operatorname{LS}\left\{\mathbf{g}(s)\left(\int_{0}^{s} \mathbf{g}^{2}(u) d u\right)^{\lambda-1}\right\}$.

$\mathrm{P} \mathrm{r}$ o o f. Assertion (a) follows from (30), (31), and the definition of $X_{t}^{ \pm, n, \lambda}$ and $Y_{t}^{ \pm, n, \lambda}$.

(b) It follows from (32) that, for $t \leqslant 1$,

$$
\begin{aligned}
\mathbf{E}\left(X_{t}^{ \pm, n+1, \lambda} \int_{0}^{1} \mathbf{f}(u) d X_{u}^{ \pm, n, \lambda}\right)= & \int_{0}^{1} \mathbf{f}(u)\left(I-\lambda K_{\mathbf{g}}^{*}\right) \\
& \times\left(I-(1-\lambda) K_{\mathbf{g}}^{*}\right)^{-1} \mathbf{I}_{[0, t]}(u) d u,
\end{aligned}
$$


and this implies

$$
\int_{0}^{t}\left(I-(1-\lambda) K_{\mathbf{g}}^{*}\right)^{-1} \mathbf{I}_{[0, t]}(u)\left(I-\lambda K_{\mathbf{g}}\right) \mathbf{f}(u) d u=0, \quad t \in[0,1] .
$$

This is reduced to $\left(I-\lambda K_{\mathbf{g}}\right) \mathbf{f}=0$, and (7) completes the proof.

For each $n \geqslant 0$ the processes $X^{ \pm, n+1, \lambda}$ and $Y^{ \pm, n+1, \lambda}$ are Brownian motions and

$$
\begin{aligned}
& \mathscr{F}_{t}^{X^{ \pm}} \supsetneqq \mathscr{F}_{t}^{X^{ \pm, 1, \lambda}} \supsetneqq \cdots \supsetneqq \mathscr{F}_{t}^{X^{ \pm, n, \lambda}} \supsetneqq \cdots, \\
& \mathscr{F}_{t}^{Y^{ \pm}} \supsetneqq \mathscr{F}_{t}^{Y^{ \pm, 1, \lambda}} \supsetneqq \cdots \supsetneqq \mathscr{F}_{t}^{Y^{ \pm, n, \lambda}} \supsetneqq \cdots .
\end{aligned}
$$

Moreover, we obtain an orthogonal decomposition, similar to (20), of the filtrations generated by $X^{ \pm}$and $Y^{ \pm}$:

$$
\begin{aligned}
& \mathscr{F}_{t}^{X^{ \pm}}=\bigoplus_{n=0}^{\infty} \sigma\left(\int_{0}^{t} \mathbf{g}(s)\left(\int_{0}^{s} \mathbf{g}^{2}(u) d u\right)^{\lambda-1} d X_{u}^{ \pm, n, \lambda}\right), \\
& \mathscr{F}_{t}^{Y^{ \pm}}=\bigoplus_{n=0}^{\infty} \sigma\left(\int_{0}^{t} \mathbf{g}(s)\left(\int_{0}^{s} \mathbf{g}^{2}(u) d u\right)^{\lambda-1} d Y_{u}^{ \pm, n, \lambda}\right) .
\end{aligned}
$$

Now, we look at some more relations between the natural filtrations of $X^{ \pm, n, \lambda}, Y^{ \pm, n, \lambda}, B^{n, \lambda}$, and $\widetilde{B^{n, \lambda}}$ which can be proved by a similar method as in Proposition 4.

Proposition 6. (i) For any $\lambda \in[0,1]$, all $n \geqslant 0$, and $t \geqslant 0$

$$
\mathscr{F}_{t}^{X^{ \pm, n, \lambda}} \oplus \mathscr{F}_{t}^{Y^{ \pm, n, \lambda}} \varsubsetneqq \mathscr{F}_{t}^{B^{n, \lambda}} \oplus \widetilde{F_{t}^{B^{n, \lambda}}}
$$

In addition, we have

$$
\begin{aligned}
H_{t}\left(B^{n, \lambda}, \widetilde{B^{n, \lambda}}\right)= & H_{t}\left(X^{+, n, \lambda}, Y^{+, n, \lambda}\right) \\
& \oplus \operatorname{LS}\left\{\int_{0}^{t} \mathbf{g}(s) d\left(\sqrt{\lambda} B_{s}^{n, \lambda}-\sqrt{1-\lambda} \widetilde{B_{s}^{n, \lambda}}\right)\right\}, \\
H_{t}\left(B^{n, \lambda}, \widetilde{B^{n, \lambda}}\right)= & H_{t}\left(X^{-, n, \lambda}, Y^{-, n, \lambda}\right) \\
& \oplus \operatorname{LS}\left\{\int_{0}^{t} \mathbf{g}(s) d\left(\sqrt{\lambda} B_{s}^{n, \lambda}+\sqrt{1-\lambda} \widetilde{B_{s}^{n, \lambda}}\right)\right\} .
\end{aligned}
$$

(ii) For any $\lambda \in[0,1]$ and $t \geqslant 0$,

$$
\mathscr{F}_{t}^{X^{ \pm, n, \lambda} \pm \sqrt{\lambda /(1-\lambda)} Y^{ \pm, n, \lambda}}=\mathscr{F}_{t}^{B^{n, \lambda} \pm \sqrt{\lambda /(1-\lambda)} \widetilde{B^{n, \lambda}}} .
$$

(iii) For all $0 \leqslant n<m$, the $\sigma$-algebra $\mathscr{F}_{t}^{X^{ \pm, n, \lambda}} \oplus \mathscr{F}_{t}^{Y^{ \pm, n, \lambda}}$ is not contained in $\mathscr{F}_{t}^{B^{m, \lambda}} \oplus \widetilde{\mathscr{F}_{t}^{B^{m, \lambda}}}$. 
$\mathrm{R}$ e $\mathrm{m}$ a $\mathrm{r} \mathrm{k}$ 7. Using representations (30) and (31) one can rewrite (33) as follows

$$
\begin{aligned}
& H_{t}\left(B^{n, \lambda}, \widetilde{B^{n, \lambda}}\right)=H_{t}\left(X^{+, n, \lambda}, Y^{+, n, \lambda}\right) \\
& \quad \oplus \operatorname{LS}\left\{\int_{0}^{t}\left(\left(I-\lambda K_{\mathbf{g}}^{*}\right)\left(I-(1-\lambda) K_{\mathbf{g}}^{*}\right)^{-1}\right)^{n} \mathbf{g}(s) d\left(\sqrt{\lambda} B_{s}-\sqrt{1-\lambda} \widetilde{B}_{s}\right)\right\} \\
& H_{t}\left(B^{n, \lambda}, \widetilde{B^{n, \lambda}}\right)=H_{t}\left(X^{-, n, \lambda}, Y^{-, n, \lambda}\right) \\
& \quad \oplus \operatorname{LS}\left\{\int_{0}^{t}\left(\left(I-\lambda K_{\mathbf{g}}^{*}\right)\left(I-(1-\lambda) K_{\mathbf{g}}^{*}\right)^{-1}\right)^{n} \mathbf{g}(s) d\left(\sqrt{\lambda} B_{s}+\sqrt{1-\lambda} \widetilde{B}_{s}\right)\right\} .
\end{aligned}
$$

$\mathrm{R} \mathrm{e} \mathrm{m} \mathrm{a} \mathrm{r} \mathrm{k}$. We will be able to treat in the same way the case $\lambda \epsilon$ $\left[0, \frac{1}{2}\right]$. For this it is enough to consider instead of $B^{\lambda}$ the process

$$
\beta_{t}^{\lambda}=\int\left(I-(1-\lambda) K_{\mathbf{g}}^{*}\right)\left(I-\lambda K_{\mathbf{g}}^{*}\right)^{-1} \mathbf{I}_{[0, t]}(u) d B_{u}
$$

which is a Brownian motion with respect to its own filtration having the property

$$
H_{t}(B)=H_{t}\left(\beta^{\lambda}\right) \oplus \operatorname{LS}\left\{\int_{0}^{t} \mathbf{g}(s)\left(\int_{0}^{s} \mathbf{g}^{2}(u) d u\right)^{-\lambda} d B_{s}\right\}
$$

Acknowledgments. The authors thank Professor Marc Yor and Professor Yuji Hibino for sending them the works [15] and [19]. The anonymous referee is acknowledged for suggestions on improving the presentation of the paper.

\section{СПИСОК ЛИТЕРАТУРЫ}

1. Alili $L ., W u C$. T. Further results on some singular linear stochastic differential equations, arxiv: math/0702785.

2. Brockhaus $O$. Sufficient statistics for the Brownian sheet. - Lecture Notes in Math., 1991 , v. 1557 , p. $44-52$.

3. Cairoli R., Walsh J. B. Stochastic integrals in the plane. - Acta Math., 1975, v. 134, p. 111-183.

4. Chaleyat-Maurel M., Jeulin T. Grossissement gaussien de la filtration brownienne. Lecture Notes in Math., 1985, v. 1118, p. 59-109.

5. Cramér $H$. On some classes of nonstationary processes. - Proceedings of the Fourth Berkeley Symposium on Mathematical Statistics and Probability, v. II. Berkeley: Univ. of California Press, p. 57-78.

6. Deheuvels $P$. Invariance of Wiener processes and of Brownian bridges by integral transforms and applications. - Stochastic Process. Appl., 1982, v. 13, № 3, p. 311318.

7. Decreusefond L., Üstünel A.S. Stochastic analysis of the fractional Brownian motion. - Potential Anal., 1998, v. 10, № 2, p. 177-214.

8. Föllmer H., Wu C.-T., Yor M. Canonical decomposition of linear transformations of two independent Brownian motions motivated by models of insider trading. Stochastic Process. Appl., 1999, v. 84, № 1, p. 137-164. 
9. Jeulin T. Semi-martingales et grossissement d'une filtration. - Lecture Notes in Math., 1980, v. 833, p. 1-142.

10. Jeulin $T$., Yor $M$. Une décomposition non-canonique du drap brownien. - Lecture Notes in Math., 1992, v. 1526, p. 322-347.

11. Jeulin $T$., Yor $M$. Filtrations des ponts browniens et équations différentielles stochastiques linéaires. - Lecture Notes in Math., 1990, v. 1426, p. 227-265.

12. Hibino $Y$. Another noncanonical representation of a Brownian motion related to singular Volterra kernels. - Proceedings of the Meijo Winter School 2003 «Quantum Information and Complexity». Ed. by T. Hida, K. Saito, and Si Si. Singapore: World Scientific, 2004, p. 202-210.

13. Hibino $Y$., Hitsuda $M$. Canonical property of representations of Gaussian processes with singular Volterra kernels. - Infin. Dimens. Anal. Quantum Probab. Relat. Top., 2002 , v. 5, № 2, p. 293-296.

14. Hibino $Y$., Hitsuda $M$., Muraoka $H$. Construction of noncanonical representations of a Brownian motion. - Hiroshima Math. J., 1997, v. 27, № 3, p. 439-448.

15. Hibino $Y$. Noncanonical representations of a multi-dimensional Brownian motion. Quantum Information and Computing. Ed. by L. Accardi, M. Ohya, N. Watanabe. Singapore: World Scientific, 2006, p. 181-185. (QP-PQ: Quantum Probability and White Noise Analysis, v. 19.)

16. Hida T. Canonical representation of Gaussian processes and their applications. Mem. Coll. Sci. Univ. Kyoto, 1960, v. 33, p. 109-155.

17. Lévy $P$. Fonctions aléatoires à corrélation linéaire. - Illinois J. Math., 1957, v. 1, p. 217-258.

18. Meyer P.-A. Sur une transformation du mouvement brownien due à Jeulin et Yor. Lecture Notes in Math., 1994, v. 1583, p. 98-101.

19. $W u$ C.-T., Yor M. Linear transformation of two independent Brownian motions and orthogonal decompositions of Brownian filtrations. - Publ. Mat., 2002, v. 46, №1, p. 237-256.

20. Yor M. Some Aspects of Brownian Motion. Part I. Some Special Functionals. Basel: Birkhäuser, 1992, 136 p. (Lectures in Mathematics ETH Zürich.)

21. Yor M. Some Aspects of Brownian Motion. Part II. Some Recent Martingale Problems. Basel: Birkhäuser, 1997, 144 p. (Lectures in Mathematics ETH Zürich.) 